\title{
Limitations of empirical supercell extrapolation for calculations of point defects in bulk, at surfaces, and in two-dimensional materials
}

\author{
Christoph Freysoldt $\odot,{ }^{1}$ Jörg Neugebauer $\odot,{ }^{1}$ Anne Marie Z. Tan $\odot,{ }^{2,3}$ and Richard G. Hennig $\odot^{2}$ \\ ${ }^{1}$ Max-Planck-Institut für Eisenforschung, Max-Planck-Straße 1, 40237 Düsseldorf, Germany \\ ${ }^{2}$ Department of Materials Science and Engineering, University of Florida, Gainesville, Florida 32611, USA \\ ${ }^{3}$ School of Mechanical and Aerospace Engineering, Nanyang Technological University, Singapore 639798, Singapore
}

(Received 21 October 2021; accepted 17 December 2021; published 7 January 2022)

\begin{abstract}
The commonly employed supercell approach for defects in crystalline materials may introduce spurious interactions between the defect and its periodic images. A rich literature is available on how the interaction energies can be estimated, reduced, or corrected. A simple and seemingly straightforward approach is to extrapolate from a series of finite supercell sizes to the infinite-size limit, assuming a smooth polynomial dependence of the energy on inverse supercell size. In this work, we demonstrate by means of explict density-functional theory supercell calculations and simplified models that wave-function overlap and electrostatic interactions lead to more complex dependencies on supercell size than commonly assumed. We show that this complexity cannot be captured by the simple extrapolation approaches and that suitable correction schemes should be employed.
\end{abstract}

DOI: 10.1103/PhysRevB.105.014103

\section{INTRODUCTION}

Electronic-structure theory, notably within the context of density-functional theory (DFT), has become an indispensable tool to understand and improve modern materials at the atomic scale [1]. For the purpose of using well-established computer codes, single defects in crystalline materials are often modeled with the supercell approach [2-6]. For this, the defect of interest and a small region of bulklike material surrounding the defect are placed in a supercell and subject to periodic-boundary conditions $[2,3]$. This approach replaces the single defect by a periodic array of defects and has several important advantages: Periodic-boundary DFT codes have been in use for decades and been shown to be accurate, reliable, and efficient; the unperturbed crystalline host material is described very accurately; periodic-boundary conditions avoid the appearance of artificial surfaces that may interact with the defect in unwanted ways, not least by truncating the host materials' screening response for electronic, electrostatic, and elastic interactions. By making the surrounding bulk region and hence the supercell sufficiently large, the limit of an isolated defect can, in principle, be recovered. In practice, one is most often limited to supercell sizes where the defect-defect interactions are still significant. A rich literature is available on how the interaction energies can be estimated, reduced, or corrected for (see, e.g., Refs. [4,7-26] as a certainly incomplete set of examples).

Published by the American Physical Society under the terms of the Creative Commons Attribution 4.0 International license. Further distribution of this work must maintain attribution to the author(s) and the published article's title, journal citation, and DOI. Open access publication funded by the Max Planck Society.
Despite recent progress in correcting the immediate results from a DFT supercell calculation by an estimate of the dominant contributions extracted from simplified models $[9,15,19$ 26], it seems still common practice and very appealing to simply compute the formation energy in several different supercells, plot them as a function of the inverse cell size (typically the linear dimension $L$, the volume $\sim L^{3}$ is used occasionally), and extrapolate to $1 / L \rightarrow 0$ using a low-order polynomial $[10,12,27]$. This is motivated by the observation that Coulomb interactions in a homogeneous system asymptotically scale like $L^{-1}$ for nominally charged systems [8] and like $L^{-3}$ for dipolar electrostatic [8] or elastic interactions [26] as well as for the alignment term of charged defects $[9,15]$. A systematic expansion in powers of $L^{-1}$ is therefore asymptotically correct.

In this work, we demonstrate that this type of extrapolation can be misleading when additional finite-size effects come into play. We consider two of these in particular. The first aspect is the ubiquitous quantum-mechanical coupling of defect wave functions, which shows the typical exponential decay of electronic effects in insulating materials $\left(e^{-\gamma L}\right)$. This is obviously good for brute-force supercell convergence, but very bad for extrapolation in inverse powers of $L$. We investigate in some detail the underlying mechanism via a tight-binding model (Secs. III A-III C) in order to identify the key factors that determine the magnitude of formation energy errors. Then, we demonstrate that the mechanistic insights explain the scaling trend in DFT calculations, taking substitutional defects in bulk diamond as an example (Sec. III D).

The second aspect we consider are electrostatic interactions in inhomogeneous systems. This aspect becomes relevant in repeated slab systems employed for defects at surfaces, interfaces, and in two-dimensional (2D) materials [19-23]. In Sec. IV, we therefore investigate the key aspects using a simplified model based on electrostatic continuum 
theory. We show how the interplay of slab thickness, cell aspect ratio, and positioning of the charge within the slab gives rise to a surprisingly rich phenomenology that often evades uniform cell-size scaling.

Last, we show that both aspects occur simultaneously when studying 2D materials. We show in Sec. V that when applying charged-defect corrections, size converged results can be obtained at supercell sizes that can be directly computed. These corrections thus remove any need for extrapolation approaches.

\section{COMPUTATIONAL DETAILS}

\section{A. One-dimensional tight-binding model}

To develop a mechanistic understanding of formation energy errors due to wave-function overlap in Secs. III B and III C, we have studied a one-dimensional (1D) tight-binding model that highlights the key mechanisms of defect state formation, band dispersion, and sampling errors. For a defect state in the band gap, the spatial decay of the electronic wave function away from its center is dictated mostly by the energetic vicinity to the nearest band edge. Therefore, our model focuses exclusively on the interplay of a single defect state with a single bulk band. For example, think of an empty defect state slightly above the valence band, where we entirely ignore the presence of a conduction band at much higher energies.

The model is described in detail in Appendix A. In this model, we consider a periodic chain of sites with a single orbital per site. Each orbital couples to its immediate neighbors. All but one site are identical: These represent the bulk. A single "defect" site has a different onsite energy and a different coupling to its neighbors. As shown in the Appendix, this model is able to produce a defect state outside the bulk band that decays exponentially away from the defect site. The implementation is available as an add-on (sx1dtb) in the SPHINX package [28].

\section{B. DFT calculations}

The DFT calculations discussed in Secs. III D and V are performed in the local-density approximation (LDA) with a plane-wave basis set and norm-conserving pseudopotentials as implemented in the SPHINX code [29]. The usual approach for obtaining formation energies from supercell setups was followed (see Ref. [4]).

For defects in diamond (see Sec. III D) a plane-wave cutoff of 40 Ry was used. Supercells are based on the cubic 8 -atom conventional unit cell. For the diamond supercells, an off-center $3 \times 3 \times 3 \mathbf{k}$-point sampling was used, with an off-center shift of $\left(\frac{1}{2}, \frac{1}{2}, \frac{1}{2}\right)$. Substitutional defects and the vacancy are located at the origin, enforcing the full local tetrahedral symmetry. Atomic geometries are not relaxed to exclude elastic interactions and Jahn-Teller distortions.

For the substitutional oxygen impurity $\mathrm{O}_{\mathrm{B}}$ in h-BN (see Sec. V), the cutoff energy was 50 Ry. The k-point sampling was adapted to the supercell size, but equivalent to at least a $16 \times 16$ sampling in the 2 -atom hexagonal unit cell, with a relative offset of $\frac{1}{2}$ in the plane and $\frac{1}{4}$ out of plane to minimize coupling across different slabs. The total cell height was kept at 40 bohrs, but we tested that other vacuum thicknesses do

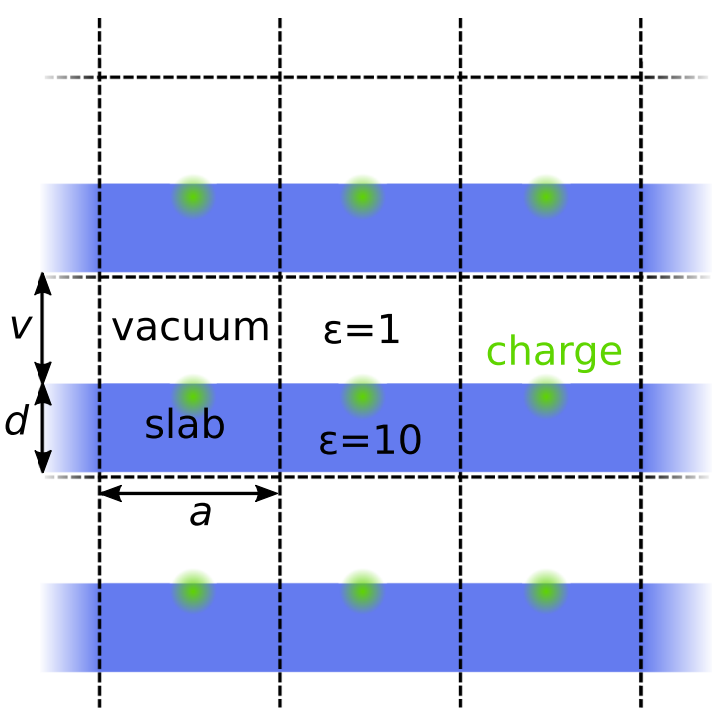

FIG. 1. Sketch of the repeated-slab approach to model charged defects (green blob), visualized as a continuum model. The dashed lines indicate the supercells. Key system parameters are the slab thickness $d$, the vacuum thickness $v$, and the lateral lattice constant $a$.

not give different results after charge correction. For the h-BN calculations, atomic positions were fully relaxed to include ionic screening, while the lateral lattice constant was kept at its theoretical value for defect-free sheets of 4.67 bohrs. Formation energies for $\mathrm{O}_{\mathrm{B}}$ in h-BN are calculated with respect to the $\mathrm{N}_{2}$-rich, $\mathrm{O}_{2}$-rich limit $\left[\mu_{\mathrm{N}}=\frac{1}{2} \mu\left(\mathrm{N}_{2} @ T=0 \mathrm{~K}\right)\right.$, $\left.\mu_{\mathrm{O}}=\frac{1}{2} \mu\left(\mathrm{O}_{2} @ T=0 \mathrm{~K}\right)\right]$, with the Fermi level at the valence band maximum of the defect-free system.

\section{Electrostatic interactions in repeated-slab systems}

For electrostatic interactions in repeated-slab systems, we use a continuum model with a $z$-dependent permittivity $\epsilon(z)$ as implemented in the sxdefectalign2d tool [22]. The associated Poisson equation is solved with the image-charge method, and allows the use of three-dimensional (3D) periodic boundary conditions or open boundary conditions on the same footing (similar to Refs. [21,23]). The difference in energy between the system under the two boundary conditions corresponds to the finite-size error of the repeated-slab approach. The dielectric model is sketched in Fig. 1. It consists of a slab of thickness $d$ and permittivity $\epsilon$ inside a periodic supercell. Inside the slab, or at its surface, there is a Gaussian charge $\left(e^{-r^{2} / 2 \beta^{2}}\right.$ with $\beta=0.5$ bohrs $)$. The key parameters of the supercell are the vacuum thickness $v$ and the lateral supercell size $a$. At fixed $(v+d) / a$ ratio, the supercell size is characterized by a single size parameter.

For all calculations in Sec. IV, the charge was set to +1 atomic charge units and the permittivity to $\epsilon=10$, a typical magnitude for semiconductors [e.g., Si: 11.7, GaN: 8.9, SiC: 9.6-10 (depending on allotrope), GaAs: 12.9]. The slab thickness was varied as indicated below. The charge was either located in the center of the slab, or at the nominal slab surface (top position). The cutoff for plane-wave expansion was set to $30 \mathrm{Ry}$. 
The sxdefectalign2d tool was also used for the defect corrections in Sec. V. As the long-range Coulomb field of a localized charge is dominated by the monopole, the precise distribution of charge within a localized defect does not need to be modeled very accurately. It is therefore common and recommended practice to use a narrow Gaussian as a charge model (standard approach), even though defectadapted models can be used to model non-Gaussian [9] or strongly anisotropic distributions [22]. If nuclear charges do not play a role, a charge distribution model can also be directly extracted from DFT electron densities [16]. As we will show in Sec. V, the use of such nonstandard, adapted models can qualitatively change the post-correction scaling behavior in 2D materials.

\section{SCALING BEHAVIOR OF WAVE-FUNCTION OVERLAP ERRORS}

\section{A. Defect states from a tight-binding perspective}

The unavoidable quantum-mechanical coupling of a defect with its periodic image via wave-function overlap leads to systematic errors in the computed defect formation energy at finite supercell size. As we will show, upon increasing the supercell size $N$, the errors reduce approximately exponentially (like $e^{-\lambda N}$ ), and hence are not well captured by commonly employed empirical "scaling laws," which are polynomials in $N^{-1}$.

The defect formation energy error is intimately linked to the band dispersion of defect-related states in the band gap. Such states are partially localized on the defect site, but exhibit tails that arise from the coupling of the bulklike bands with localized defect orbitals. In the limit of an isolated defect (infinite supercell size), each such state has a well-defined energy. For finite supercells, however, this single state develops into a dispersive band, i.e., the energy varies with the $\mathbf{k}$ point within the irreducible Brillouin zone of the supercell. Not surprisingly, the magnitude of the formation energy error depends on the k-point sampling scheme and, if the band is partially occupied, also on the occupation scheme (FermiDirac smearing vs constant occupations).

The defect energy modifications do not directly result from the coupling of the defect states per se, but occupation differences play an essential role. Think of the standard textbook model of quantum mechanical bond formation from the overlap of two orbitals at energies $\epsilon_{A}$ and $\epsilon_{B}$. Let us assume they have initial occupations $f_{A}$ and $f_{B}$, respectively, which can be empty $(f=0)$ or filled $(f=1)$. When brought into contact, the orbitals form a bonding combination at $\epsilon_{1}=\frac{\epsilon_{A}+\epsilon_{B}}{2}-\Delta E$ and an antibonding combination at $\epsilon_{2}=\frac{\epsilon_{A}+\epsilon_{B}}{2}+\Delta E$ (with $\Delta E \geqslant 0$ ). The electronic energy $E=f_{1} \epsilon_{1}+f_{2} \epsilon_{2}$ generally differs from the one for separate orbitals $\left(f_{A} \epsilon_{A}+f_{B} \epsilon_{B}\right)$ unless all occupations are the same, i.e., $f_{1}=f_{2}=f_{A}=f_{B}$. Thus, the mere coupling of either empty or filled defect states would not change the energy in this simple picture. For partially filled states, however, there is a primary overlap effect if the electrons are redistributed according to a Fermi-Dirac distribution or any other smearing scheme (such as Gaussian, Methfessel-Paxton, ...). Without countermeasures or corrections, the formation energies from smeared occupations strongly vary with $\mathbf{k}$-point sampling and supercell size [4]. Obviously, the primary overlap effect can be suppressed easily by setting the occupations of the defect band to a constant value throughout the Brillouin zone. Yet, there is also a secondary overlap effect that cannot be suppressed and occurs even for completely empty or filled defect states: If the localized defect orbital (occupation $f_{d}$ ) couples to a bulk band with a different occupation $f_{b} \neq f_{d}$ (i.e., an empty defect state to the filled valence band, or a filled defect state to the empty conduction band), the overlap of defect wave-function tails may modify the coupling between bulk state and defect orbitals, and hence also the total energy.

To develop the argument, we will first study this for a one-dimensional (1D) tight-binding model that highlights the key mechanisms of defect state formation, band dispersion, and sampling errors. Later, in Sec. III D, we will also present DFT calculations for neutral defects in diamond, demonstrating that the insights from the tight-binding model are transferable to 3D bulk supercells. The 1D model described in Appendix A has an analytic solution for the isolated limit. This analytic solution is the main reason why we restrict ourselves to a one-dimensional model. A generalization of the supercell model to three dimensions, i.e., on a cubic lattice, is straightforward, but we were unable to find an analytic solution for the isolated limit in this case. When studying the numerical behavior of the 3D analog of the tight-binding model (not presented here), we also observed a more complex $e^{-\left[\lambda_{\infty}+\Delta \lambda(N)\right] N}$ scaling behavior of the supercell dispersion, where $\Delta \lambda(N)<\lambda_{\infty}$ rapidly and monotonously goes down to zero. In other words, the true scaling behavior in three dimensions is rapid, yet only approximately exponential for supercell sizes accessible within DFT.

\section{B. Defect band dispersion}

Coming back to the one-dimensional version, it is the energetic position of the defect state relative to the bulk band edge that directly determines the spatial decay of the wave function, and hence also how quickly the defect band dispersion becomes smaller with respect to supercell size. Yet, the magnitude of dispersion also depends on the coupling strength between the defect site and its neighbors. Figure 2 shows a zoom-in of the defect band for three different coupling strengths $t_{d}$ at $N=5$. In the isolated-defect limit, all three cases show the same energy $E_{d}=0.2 \mathrm{eV}$, and the corresponding wave-function decay rate $\gamma$ in the isolated limit is the same. However, since the weight of the tail region increases with coupling strength, the strong coupling case also shows a significantly enhanced defect-defect interaction compared to the weakly coupling case. In consequence, the position of the defect state within the band gap alone does not suffice to estimate the amplitude of band dispersion.

One can also clearly see that the dispersion is not symmetric around the isolated defect energy, but enhanced for energies below it. Also, the defect band crosses the isolated limit not at the Brillouin zone center $\left(k=\frac{\pi}{2}\right)$, but where $\cos k \approx e^{-\gamma N}$ (This condition arises from perturbation 


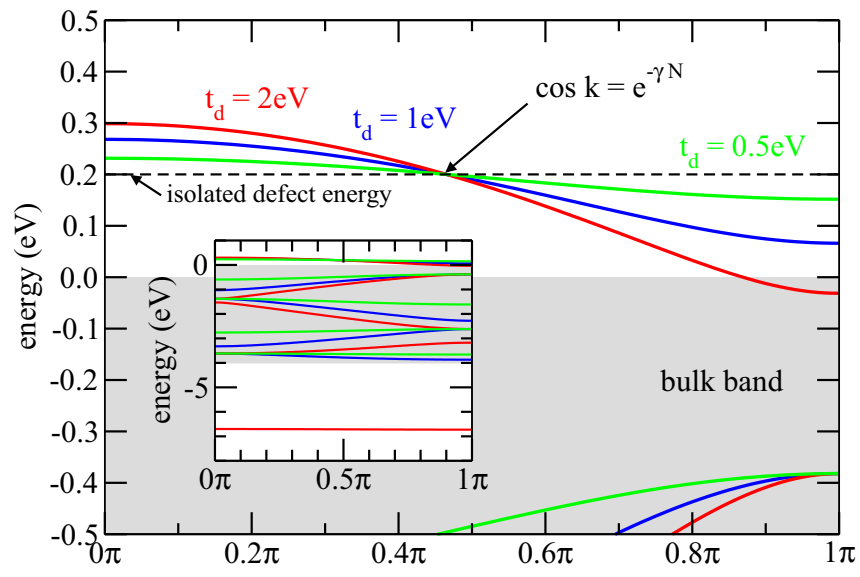

FIG. 2. Defect band dispersion in the tight-binding model $(N=$ $5, t_{b}=1 \mathrm{eV}, E_{d}=0.2 \mathrm{eV}$ ) for three coupling strengths: $t_{d}=2 \mathrm{eV}$ (red), $t_{d}=1 \mathrm{eV}$ (blue), and $t_{d}=0.5 \mathrm{eV}$ (green). The inset shows an overview over the entire energy range. The energy range of the bulk host band is shown in gray. For the crossing point with the isolated limit, see text.

theory. ${ }^{1}$ ) The band dispersion asymmetry arises from the dependence of the spatial decay constant on distance from the band edge: For energies closer to the band edge, the defect state decays more slowly and hence couples more strongly to its periodic images. In consequence, the average defect band energy is systematically below its isolated limit. We will investigate in the next section how this effect translates to errors in the calculated formation energies.

\section{Finite-size errors for different k-point sampling schemes}

The error in total energies is closely related to the error in the occupation-weighted average defect state energy (see Appendix B). This average depends, in an actual defect calculation, on the Brillouin zone sampling and defect state occupation scheme. The finite-size error in the defect formation energy arising from the electronic band energy is [cf. Eq. (B5)]

$$
\Delta E^{f}=\sum_{\mathbf{k}} w_{\mathbf{k}}\left(f_{d \mathbf{k}}-f_{b}\right)\left(\epsilon_{d \mathbf{k}}-E_{d}\right),
$$

where $w_{k}$ is the integration weight, and $f_{d \mathbf{k}}$ and $\epsilon_{d \mathbf{k}}$ are the occupation and defect state eigenvalue for each $\mathbf{k}$ point, respectively. $E_{d}$ is the defect state energy in the isolated limit. Additional density-related terms are expected to show

\footnotetext{
${ }^{1}$ If one constructs the periodic wave function by a phase-adapted superposition of the isolated states, one can estimate the energy to be given by

$$
\epsilon(k)=E_{d}+H_{\text {eff }} \sum_{n=1}^{\infty} e^{-(\gamma N+i k) n}+e^{-(\gamma N-i k) n},
$$

where $H_{\text {eff }}$ parametrizes the perturbation energy of the defect tail due to the presence of the other defect sites at chain position $n N$, where the corresponding decay and phase factors appear in the sum. The sum can be evaluated analytically (geometric series), and setting it to zero yields the condition mentioned in the text.
}

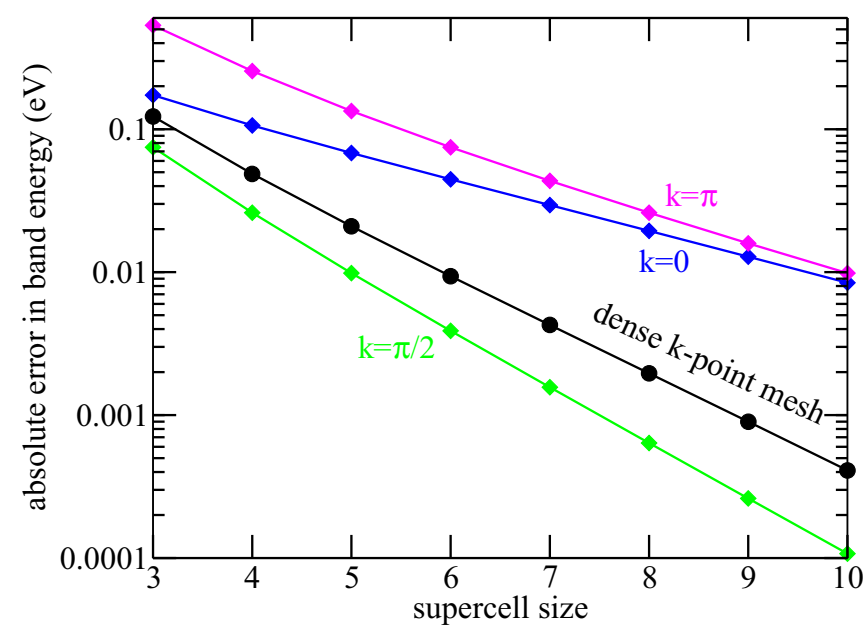

FIG. 3. Absolute errors in defect band energy of the tight-binding model $\left(t_{d}=1 \mathrm{eV}\right)$ with different $k$-point summation schemes: Special $k$ points at $0, \frac{\pi}{2}$, and $\pi$, converged averages (100k points) for constant occupation. All but one error have negative sign ( $\bar{\epsilon}$ below $E_{d}$ ), the exception is $k=0$ (maximum of the defect band).

an exponential convergence as the defect-induced density is coupled to the exponentially decaying defect states. For constant occupation $\left(f_{d \mathbf{k}}=f_{d}\right)$, the equation simplifies to

$$
\Delta E^{f}=\left(f_{d}-f_{b}\right) \sum_{\mathbf{k}} w_{\mathbf{k}}\left(\epsilon_{d \mathbf{k}}-E_{d}\right) .
$$

We will therefore focus on different k-point samplings in the following. Makov et al. proposed to use special $\mathbf{k}$ points to minimize defect-defect interactions [7]. In our case, this would correspond to $k=\frac{1}{2} \pi$. At this point, the contribution from the direct images at $i=2 \pm N$ vanishes, but those of the images at $i=2 \pm 2 N$ lead to a small systematic underestimation. In Fig. 3, we compare the convergence of different Brillouin zone sampling schemes with supercell size. These are the defect band energy at $k=0, \frac{\pi}{2}$, and $\pi$ as well as the Brillouin zone average (100 $k$ points) with equal weights. The model parameters are as before $\left(t_{b}=t_{d}=1 \mathrm{eV}\right.$, $\left.E_{d}=0.2 \mathrm{eV}\right)$.

All k-point samplings show a band energy error that decays approximately exponentially with increasing supercell size. The most striking difference is in the slope in the logarithmic plot: While the $k=0$ and $k=\pi$ cases pick up the contribution from the nearest images, these contributions cancel for the dense $k$-point mesh and the $k=\frac{\pi}{2}$ case. Therefore, these latter cases converge about twice as quickly compared to the former ones. We note in passing that $k=0$ corresponds to $\Gamma$ only sampling, which is a particularly bad choice for avoiding wave-function overlap related errors even for large supercells [4]. The best option for this simple model is the $k=\frac{\pi}{2}$ special point, followed by the constant occupation scheme, which, other than $k=\frac{\pi}{2}$, picks up the entire dispersion asymmetry throughout the Brillouin zone. Thus, special k-point sampling that avoids the extrema of the defect band is expected to be superior when feasible, but of course restricts the k-point sampling of the host material to a supercell-specific sampling mesh. In general, it is best to employ a homogeneous, sufficiently dense k-point sampling and use constant occupations to largely average out the band dispersion. 


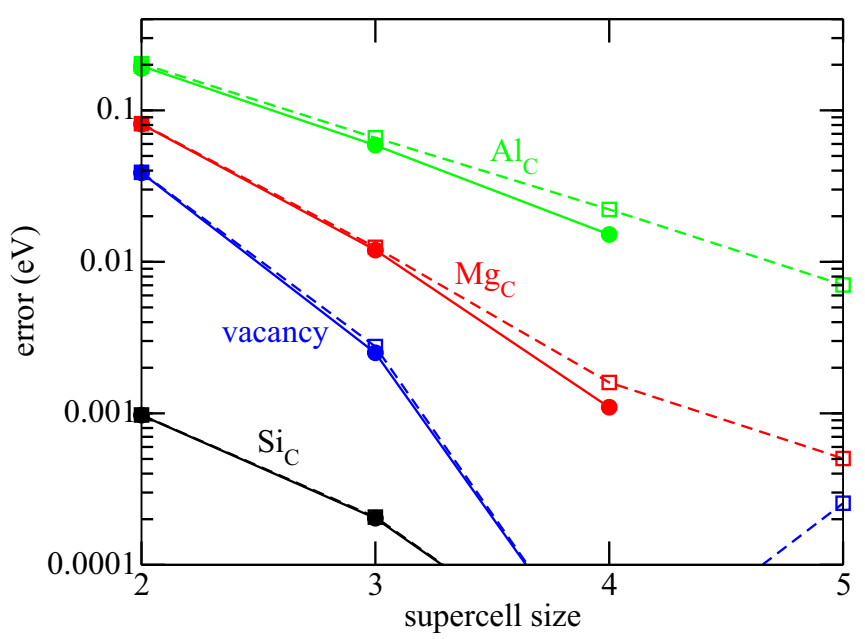

FIG. 4. Estimated supercell errors $\left|E^{f}(N)-E^{f}(\infty)\right|$ for chargeneutral defects in diamond from DFT-LDA $N \times N \times N$ supercells. The lines are guides to the eye. Solid lines and circles: $E^{f}(\infty) \approx$ $E^{f}(N=5)$. Dashed lines and empty squares: $E^{f}(\infty)$ from extrapolated value from exponential fit [see Eq. (3)].

The key insights of the tight-binding model can be summarized as follows. Defect states in the band gap may arise from the coupling of localized defect orbitals with the host bands. Their spatial decay is dictated by the energetic difference from the band edge, and this qualitatively determines an approximately exponential decay of band dispersion effects with increasing supercell size. The magnitude of dispersion, however, depends not only on the defect state's energy, but also on the coupling strength between the defect orbital and its neighbors. If the defect band is occupied differently than the host band it couples to, wave-function overlap effects cause errors in the formation energy that scale with the occupation difference. Band dispersion is asymmetric and can therefore not completely be removed by using a constant occupation scheme and special or systematic k-point sampling. Yet, schemes that aim at reducing the dispersion effects by cancellation are helpful and significantly increase the speed of convergence.

\section{Finite-size errors from wave-function overlap: DFT results}

Before we come to the consequences for $1 / N$ supercell extrapolation, we shall briefly compare the qualitative model predictions with real DFT calculations. For this, we compare in Fig. 4 the convergence behavior of the formation energy of four point defects in diamond: The charge-neutral $\mathrm{Mg}, \mathrm{Al}$, and Si substitutional impurities, and the vacancy. To exclude strain effects, atomic geometries were not relaxed. For simplicity, spin was not considered explicitly, so the occupation numbers include the spin multiplicity and can vary between 0 (empty) and 2 (fully occupied). These defects exhibit a threefold defect band at varying depth in the band gap, which was occupied equally with $f_{d}=\frac{2}{3}$ (vacancy), $\frac{4}{3}(\mathrm{Al}), \frac{5}{3}(\mathrm{Mg})$, and $2(\mathrm{Si})$, respectively. While the Si state merges with the bulklike bands for all supercells used here, the other cases have a defect band at $\sim 0.03 \mathrm{eV}(\mathrm{Al})<0.4 \mathrm{eV}(\mathrm{Mg})<1.5 \mathrm{eV}$ (vacancy) above the valence band maximum of bulk diamond. Correspondingly,

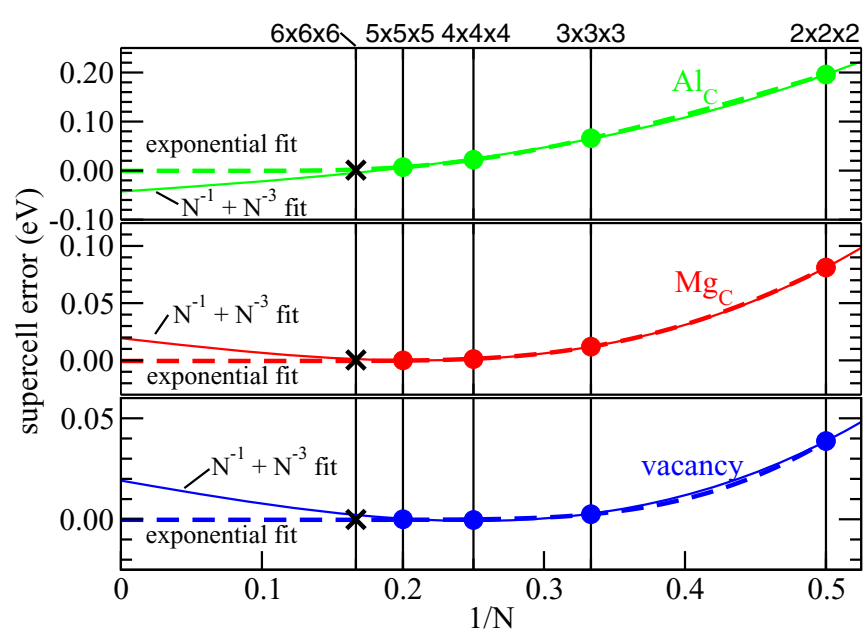

FIG. 5. $1 / N$ plot of estimated supercell errors $(N \times N \times N)$ for charge-neutral defects in diamond from DFT-LDA. The solid lines are fits with $N^{-1}$ and $N^{-3}$ terms. For $\mathrm{Mg}_{\mathrm{C}}$ and the vacancy, the $N=$ 5 result was taken as the converged value. For $\mathrm{Al}_{\mathrm{C}}$, the converged value was estimated from an exponential fit (see text). Exponential fits (dashed lines) are shown for comparison. Black crosses show control calculations performed for a $6 \times 6 \times 6$ supercell.

the defect spatial decay constant increases. For $\mathrm{Si}$, we do not expect a significant error from wave-function overlap as $f_{d}=f_{b}$. Indeed the calculated formation energies of $\mathrm{Si}_{\mathrm{C}}$ for all supercell sizes agree to within $1 \mathrm{meV}$. The defects with partially occupied states show an approximately exponential convergence $[E(\infty), A, \lambda$ are fitting parameters) $]$

$$
E(N) \approx E(\infty)+A e^{-\lambda N},
$$

with a varying convergence rate $\lambda$ (slope), as expected from the tight-binding model.

What happens if we extrapolate these errors to infinite size using polynomials in $N^{-1}$ ? Figure 5 shows the same data as in Fig. 4, but plotted against $1 / N$ together with least-square fits (solid lines) to the physically inappropriate, but Coulombinspired form

$$
\Delta E=C_{0}+C_{1} N^{-1}+C_{3} N^{-3}
$$

for all but $\mathrm{Si}_{\mathrm{C}}$ which has errors $\leqslant 0.001 \mathrm{eV}$ within our numerical noise limit. For $\mathrm{Mg}_{\mathrm{C}}$ and the vacancy, we have used the $N=5$ result as an estimate for the converged values. For $\mathrm{Al}_{C}$, we have used, based on the insight that the error scales approximately exponentially, a fit to Eq. (3) to estimate the remaining error beyond the $5 \times 5 \times 5$ supercell, which suggests that the converged value lies $0.007 \mathrm{eV}$ below the $N=5$ value (cf. green squares in Fig. 4). Control calculations for $N=6$, that were not used for the fitting, are shown as black crosses.

The quality of the fits looks very convincing. When ignoring the physical background, it would appear plausible that they describe well the supercell dependence of the error. In the usual extrapolation schemes the fitted functions are used to estimate the isolated-defect limit via the value at $1 / N \rightarrow 0$ (which should be zero in our plot). Figure 5 clearly demonstrates that the extrapolation errors are significant compared to the errors of all input data; in all three cases the second-worst $N=3$ value without further extrapolation is about as accurate 
as the $N^{-1}+N^{-3}$ extrapolated one. That is, extrapolating makes the accuracy much worse than just taking the value from the largest supercell. We have also tested other polynomial forms, for instance, including an unphysical $N^{-2}$ term, or focusing exclusively on the $N^{-1}$ term. These alternative expressions lead to different numbers, but similar magnitudes (not shown). For the present case, this poor performance comes not as a surprise [there is no justification for using Eq. (4), and therefore, one cannot expect the fit to be helpful]. However, and that is the main point of this entire discussion, wave-function overlap related errors with an exponential convergence behavior are ubiquitous in defect calculations, namely, whenever the occupation of a defect-related state in the band gap differs from that of the host band that mediates the defect band dispersion. Supercell extrapolation aiming at Coulomb interactions and other functional dependencies will systematically magnify the wave-function overlap related errors.

\section{SCALING OF ELECTROSTATIC INTERACTIONS IN REPEATED-SLAB SYSTEMS FROM A DIELECTRIC CONTINUUM MODEL}

Electrostatic interactions introduce significant artifacts in the calculation of formation energies of charged defects at surfaces or in 2D materials within the repeated-slab approach. This is not only due to the reduced screening in vacuum, but also because the lateral size (area of the surface unit cell) and the surface-normal direction show a qualitatively different convergence behavior. If the surface unit cell is kept fixed, an increase in the vacuum spacing leads to an asymptotically linear increase in the formation energy due to the capacitor effect $[22,30]$. At fixed vacuum thickness, on the other hand, an increase of the surface unit cell leads to a logarithmic divergence [31,32]. Only increasing all dimensions simultaneously guarantees convergence. ${ }^{2}$ Of course, additive correction schemes are available as a practical and, in our view, better alternative $[20,22,23]$. Interestingly, scaling and extrapolation are still ingredients of schemes that lack a direct calculation of the isolated case [20], and these extrapolations can be misleading [33]. The purpose of this section is to demonstrate that the phenomenology of scaling may be surprisingly complex, and that it is not well captured by the commonly used low-order polynomials in the inverse scaling parameter.

To this end, we computed the uniform supercell scaling behavior within dielectric continuum theory using the sxdefectalign2d tool [22]. We consider a number of prototypical cases with a tetragonal $a \times a \times c$ repeated-slab cell. These cases differ in

(1) the slab thickness $d$ (this remains unscaled),

(2) the position of the defect within the slab [center (c) or surface $(t=$ top $)$,

\footnotetext{
${ }^{2} \mathrm{~A}$ simple argument for the scaling behavior is this: In the reciprocal space formulation for a $D$-dimensional scaling, the decisive term has the form $\int_{0}^{g_{\text {cut }}} d g g^{3-D}$, which must be evaluated at a boundary $g_{\text {cut }} \approx 1 / L$, yielding scalings of $L, \ln (L)$, and $L^{-1}$ for $D=1,2$, and 3 , respectively.
}
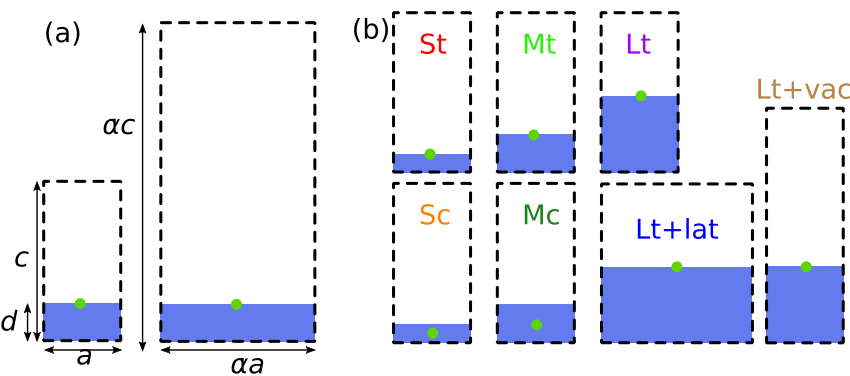

FIG. 6. Supercell geometries for slabs. (a) Scaling of supercell, but not of the slab thickness. (b) Supercell and slab geometries used in Fig. 7. $S=$ small slab thickness, $M=$ medium slab thickness, $L=$ large slab thickness. Defect position within the slab is " $t$ " (top) or " $c$ " (centered). Special cases +lat $=$ laterally extended, and + vac $=$ extra vacuum.

(3) the vacuum thickness $v=c-d$ in the smallest cell, and

(4) the aspect ratio $c / a$ of the repeated-slab cell.

Upon scaling, the lateral size and the total cell height (vacuum + slab) are increased by the same factor $\alpha$, while the slab thickness is kept constant [see Fig. 6(a)]. In practice, lateral scaling must be commensurate to the underlying lattice. In addition to integer numbers, we also include $\sqrt{2}$ (as realized in a c $2 \times 2$ cell $), \sqrt{5}$ [as realized in a $(1,2) \times(2,-1)$ cell], and $2 \sqrt{2}$ (a c4 $\times 4$ cell) to fill the gaps at low scaling factors. The different supercell and slab geometries are depicted in Fig. 6(b). Three possible slab thicknesses were considered: $d=5$ bohrs (small, denoted by $S$ ), $d=10$ bohrs (medium, denoted by $M$ ), and $d=20$ bohrs (large, denoted by $L$ ). The defect's position with respect to the dielectric slab can either be in the center, denoted by " $c$," or at the surface, denoted by " $t$ " (top). The initial cell size is $a \times a \times c$ with $a=20$ bohrs (except for " $L t+$ lat" with $a=40$ bohrs), and $c=40$ bohrs (except for " $L t+$ vac" with $c=60$ bohrs).

The results are shown in Fig. 7, plotted as a function of the inverse of the scaling factor $\alpha$. It is obvious that the scaling behavior can exhibit local minima and maxima and cannot be described well by a low-order polynomial fitted to a small- $\alpha$ range, as highlighted in Fig. 8.

At small scales up to $\alpha \approx 4$, the position of the charge within the slab has an important influence. Charges at the surface tend to show a stronger artificial stabilization (i.e., lower formation energies) than charges in the center. The stabilization has its root in that the Coulomb attraction between the defect charge and the implicit compensating background is larger than the repulsive interaction between the localized charge and its likewise localized periodic images. The charges in the center experience less of this due to the stronger screening inside the slab. When the lateral distance becomes large compared to the slab thickness, this influence of the charge position within the slab vanishes (see $S c$ vs $S t$ and $M c$ vs $M t$ ). The thicker the slab, on the other hand, the more pronounced the center vs surface position effect becomes. These intraslab interactions compete with the interslab capacitor effect, that depends mostly on the aspect ratio of the total cell and on the dielectric image-charge effect at small vacuum distances [34]. The total competition can lead to an almost perfect linear 


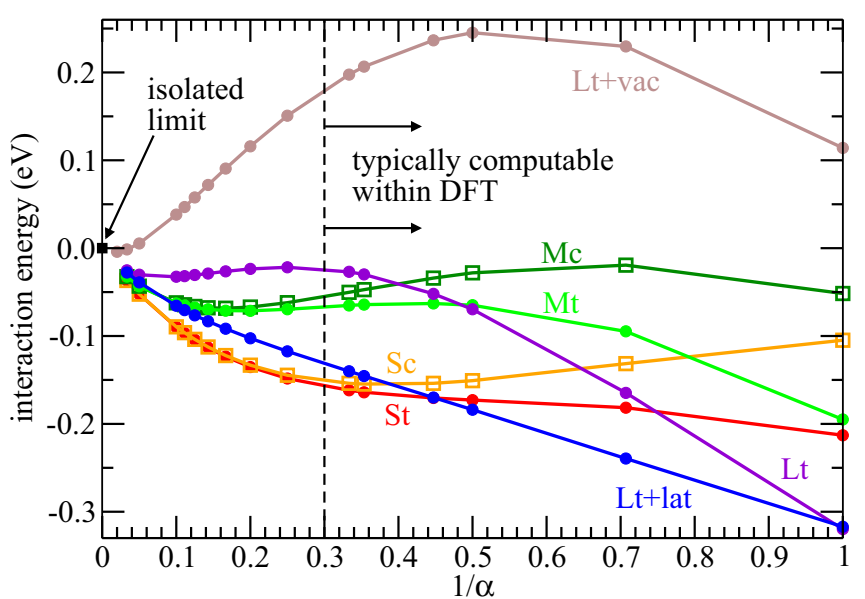

FIG. 7. Scaling behavior of electrostatic energies in slab systems from the dielectric continuum model upon uniform scaling $(\alpha)$ of cell dimensions. The formation energy of the isolated case $(\alpha \rightarrow \infty)$ has been set to zero. Slab thickness is encoded by capital letter $S$ (5 bohrs), $M$ (10 bohrs), and $L$ (20 bohrs) [cf. Fig. 6(b)]. The charge's position is encoded by lowercase letter $c$ (center of slab) or $t$ (top of slab, i.e., at the surface). Tetragonal cells $(\alpha a \times \alpha a \times \alpha c)$ are used, with typically $a=20$ bohrs (except for $L t+$ lat: $a=40$ bohrs) and $c=40$ bohrs (except for $L t+$ vac: $c=60$ bohrs).

behavior over a wide range of $1 / \alpha$, e.g., in the " $L t+$ lat" case, that "suddenly" turns up at $1 / \alpha \approx 0.18$ to approach the asymptotic limit. In other cases, rather flat regions or pronounced up-and-down behavior may occur.

The key insight of this exercise is to demonstrate that even a seemingly simple scenario can lead to rather complex global scaling behavior that is difficult to characterize from a limited $\alpha$ range assuming a global low-order polynomial shape. Extrapolation from a restricted view can therefore be highly misleading. In all cases shown above, low-order polynomial extrapolation is very sensitive to the considered range, and can lead to severe mispredictions. To illustrate

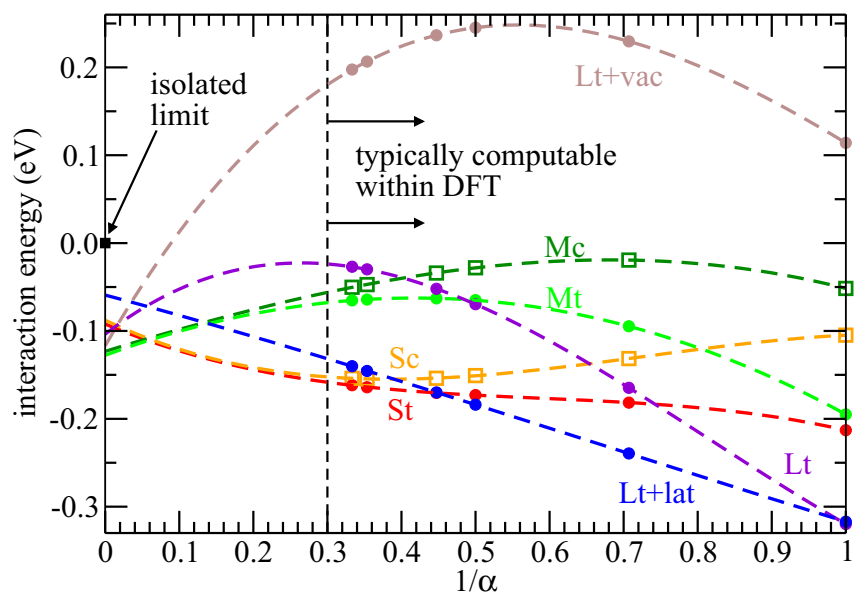

FIG. 8. Same data as Fig. 7 for $\alpha \leqslant 3(1 / \alpha \geqslant 0.33)$, with thirdorder polynomials fit to the values. Low-order polynomial fits in this region completely fail to anticipate the true asymptotic behavior (cf. Fig. 7). The formation energy of the isolated case $(1 / \alpha \rightarrow 0)$ has been set to zero.

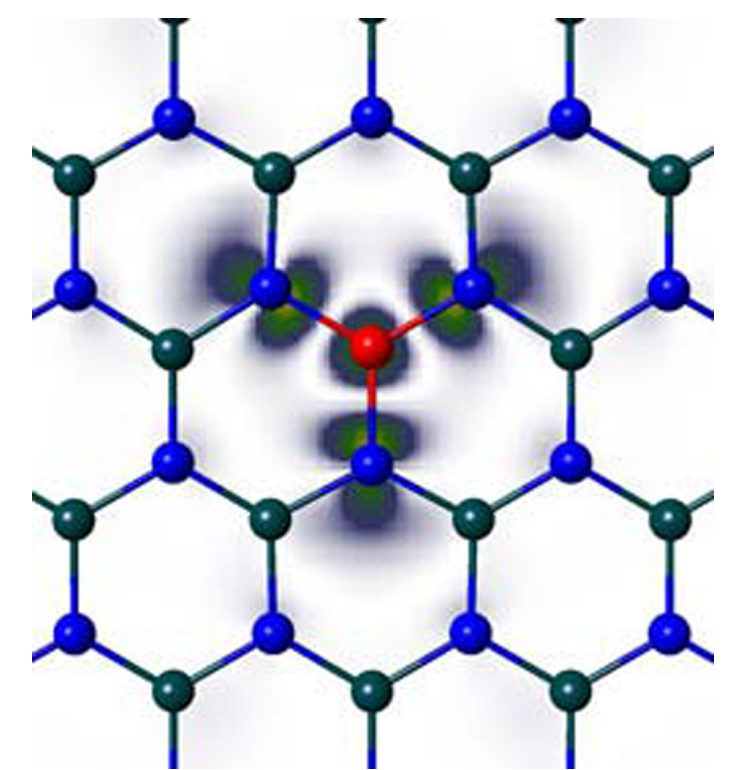

FIG. 9. Amplitude of the empty defect state of $\mathrm{O}_{\mathrm{B}}$ in h-BN, overlayed with the atomic structure (red: Oxygen, blue: Nitrogen, dark green: Boron).

this, Fig. 8 shows third-order polynomials in $1 / \alpha$ that were fitted to the small supercell data $(\alpha \leqslant 3)$ via regression. Not only do these fits systematically fail to reflect the asymptotic behavior, they also predict limiting values at $1 / \alpha \rightarrow 0$ that deviate significantly from the true value of zero. Therefore, for slab systems absolute correction schemes that directly treat the isolated-defect limit with appropriate boundary conditions should be preferred over scaling-based approaches. Such correction schemes are freely available $[22,23,35]$.

\section{POST-CORRECTION EXTRAPOLATION}

As a last example, we want to demonstrate here that even after applying corrections to the leading Coulomb errors, extrapolation is not a reliable tool to assess the importance of "further" effects, or their scaling behavior. For this we consider a charged defect in a 2D system, namely, the relaxed $\mathrm{O}_{\mathrm{B}}$ substitutional defect in a h-BN monolayer in its +1 charge state and study the convergence of the formation energy from DFT calculations. The defect was selected because it shows both electrostatic and exponential tail effects. For the latter, the defect provides an empty defect state slightly above the valence band edge. It is derived from $s$ and $p$ orbitals lying in the BN plane and can couple to the corresponding $\sigma$-type bonding orbitals inside the BN valence band (see Fig. 9). In addition, there is an occupied defect state dominated by the out-of-plane oxygen $2 p$ orbital that hybridizes with the occupied N $2 p$ valence band edge (not shown). In the absence of the $\mathrm{O}$ defect, however, the empty boron $2 p_{z}$ orbital at this site would contribute mostly to the conduction band. The presence of this state therefore induces a coupling of $\mathrm{p}_{z}$-derived occupied valence and empty conduction band states.

Without charge corrections, the computed values strongly depend on the supercell size [see the uncorrected values (magenta) in Fig. 10]. After applying standard 2D charge corrections [22] with a narrow Gaussian ( $\beta=0.5$ bohrs) as 


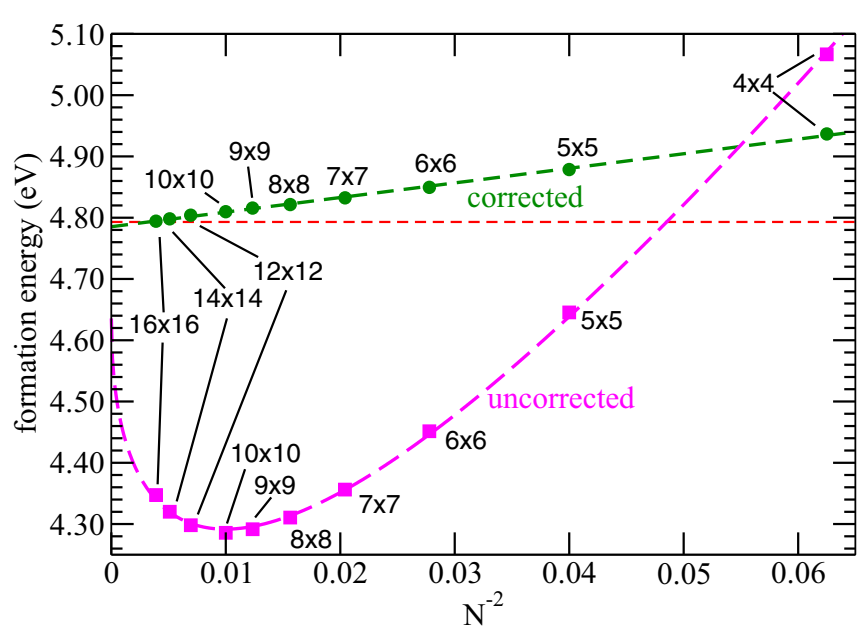

FIG. 10. Formation energies of the $+1 \mathrm{O}_{\mathrm{B}}$ defect in single-sheet h-BN, obtained from various supercells. Magenta circles: Uncorrected values. Magenta dotted line: Polynomial fit with $N^{-1}$ and $N^{-2}$ terms (unphysical). Green dots: Values including a standard 2D correction (Gaussian width 0.5 bohrs). The green dashed line shows a linear fit to the apparent $1 / N^{2}$ behavior. The red dashed line represents the value extracted from an improved procedure (see Fig. 12).

charge model, the results show a rapid convergence with respect to the vacuum size (not shown here). We therefore focus on convergence with respect to lateral size. Figure 10 shows the formation energies with and without correction for $N \times N$ lateral size from $4 \times 4$ (32 atoms) to $16 \times 16$ (512 atoms), plotted against the inverse of the unit-cell size $N^{2}$.

The uncorrected values can be easily fitted to a simple form in $N^{-1}$, leading to an extrapolated value of $4.64 \mathrm{eV}$. As we know that lateral scaling should exhibit a logarithmic divergence, this uncorrected extrapolation is misleading despite the very good fit in the accessible range.

But even after correction, there is a systematic trend: It is very apparent that the corrected formation energies carry an error that seems to scale like $1 / N^{2}$. There are several effects that should scale like $1 / N^{2}$ in a $2 \mathrm{D}$ material, such as elastic stress (see Appendix C). Likewise, defect-related excess contributions to macroscopic system properties (permittivity, bulk moduli) scale inversely with concentration, which could modify the system's long-range response to the presence of the defect. It is highly tempting to capture them all by extrapolating to $1 / N^{2} \rightarrow 0$. Doing so, the converged value would be estimated to be $4.785 \mathrm{eV}$.

Such a reasoning about the origin of the remaining $1 / N^{2}$ scaling trend would usually not arouse doubts, but turns out to be wrong and misleading. We presented this speculation here only to demonstrate how easy it is to rationalize simplelooking trends. What went wrong is this: While the charge corrections with the strongly localized Gaussian charge $(\beta=$ 0.5 bohrs) indeed remove the leading Coulomb error and the vacuum dependence, a careful analysis shows that they do overestimate the lateral screening. The reason is that the defect charge smears out laterally to the neighbor nitrogen atoms (cf. the shape of the emptied defect state in Fig. 9), and thus is much less localized than the default parame-

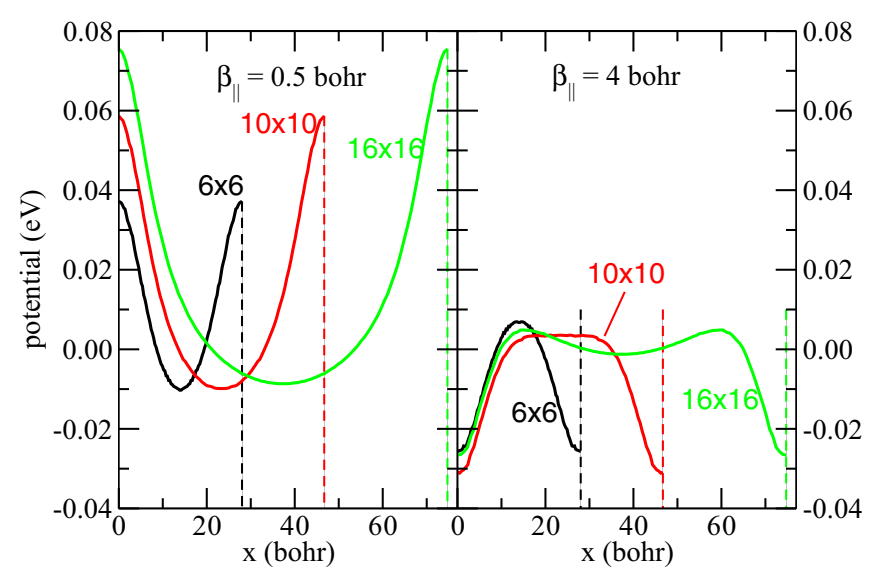

FIG. 11. Line scan of the post-correction short-range potential at $z=10$ bohrs in the vacuum above the BN slab along the $x$ direction. Left: Narrow Gaussian charge $(\beta=0.5$ bohrs $)$. Right: Improved charge model $\left(\beta_{\|}=4\right.$ bohrs). Three different cell sizes are shown: $6 \times 6$ (black), $10 \times 10$ (red), and $16 \times 16$ (red). Each scan starts above the defect at $x=0$, and ends above its periodic image (vertical dashed line).

ter assumes. This electrostatic origin leaves its traces in the corrected potential. A perfectly modeled charge distribution would yield an extended plateau in the short-range potential used in the correction scheme [9]. Different supercells beyond a certain threshold size should further agree on the shape of this short-range potential near the defect. When the potential is inspected in a full $x y$ plane within the vacuum rather than only its $x y$-averaged profile along the $z$ axis, a significant lateral variation becomes visible when the default parameters are employed (see left side of Fig. 11).

This can be fixed by adapting the lateral broadening of the model charge. When the lateral extent of the model charge is properly chosen, the lateral variation of the potential no longer increases with lateral cell size, but provides an extended plateau (right side of Fig. 11). Likewise, all cell sizes then provide similar, well-aligned shapes of the short-range potential in the vicinity of the defect. Using such an improved model with $\beta_{\|}=4$ bohrs for the correction, we recomputed the formation energies and show them in Fig. 12. All of a sudden, the previously striking $1 / N^{2}$ trend has made room to rapid convergence, that probably reflects the exponential decay of the defect states. A fit to an exponential behavior $E_{N}=E_{\infty}+A e^{-\lambda N}$ shows indeed an excellent agreement (see violet line in Fig. 12), which can be taken as a hint, but not a proof of the wave-function origin of the trend. Beyond the $6 \times 6$ cell, all results now agree to within less than $5 \mathrm{meV}$. We note in passing that we cannot expect much better results by going for even larger cells: The largest $16 \times 16$ cell has 512 atoms; to achieve a $5-\mathrm{meV}$ accuracy in the formation energy from the energy difference of two 512-atom calculations requires to keep the unavoidable numerical noise in each separate calculation to within $0.01 \mathrm{meV}$ per atom.

Additional evidence for an electronic origin of the remaining deviations for small cells comes from the excellent correlation (within $2 \mathrm{meV}$ ) between the energy level of the empty defect state (averaged over $\mathbf{k}$ points, including charge corrections) and the corrected formation energy (see the 


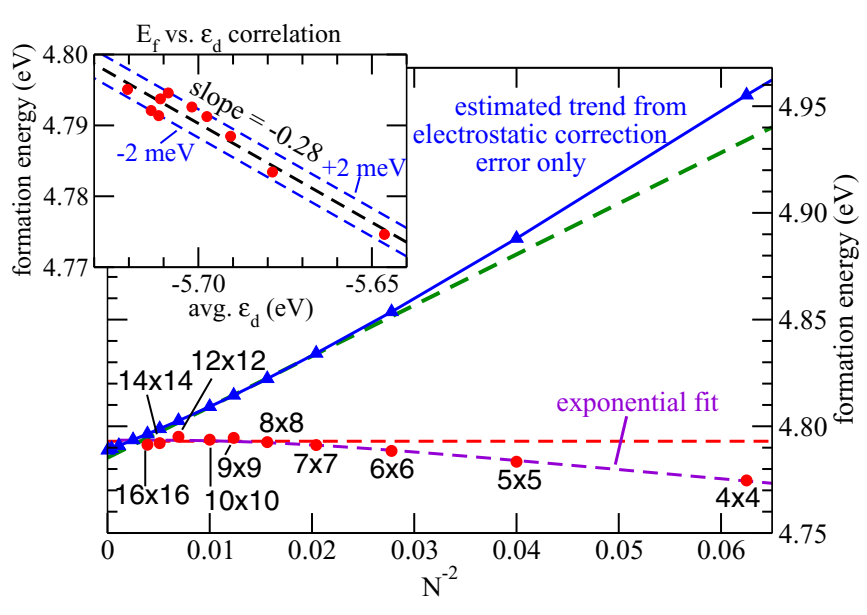

FIG. 12. Formation energies of the $+1 \mathrm{O}_{\mathrm{B}}$ defect in single-sheet h-BN, obtained from various supercells using an improved electrostatic correction (see text). The green dashed line represents the trend of Fig. 10. The violet dashed line is an exponential fit of the improved data (see text). The red dashed line depicts the estimated converged value. The blue line (triangles) depicts the estimated trend if there was only the correction error from the insufficient correction used for Fig. 10. The inset demonstrates the correlation between the average defect level and the formation energy.

inset of Fig. 12). This is in qualitative agreement with the discussions of Appendix B. Yet, the quantitative correlation $(-0.28)$ is much smaller than expected for a simple coupling of one defect orbital to a single-orbital valence band which would yield $f_{d}-f_{b}=-2$. As multiple defect orbitals couple to different bulk bands, and as not all defect states appear in the band gap and can be quantitatively analyzed, we refrain from attempting quantitative corrections for the exponential tail effects.

In this particular case, the $1 / N^{2}$ extrapolated value from Fig. 10 using a narrow Gaussian (green dashed line) agrees to within $0.01 \mathrm{eV}$ with the value extracted from the improved charge model. Indeed, the fast-decaying nonelectrostatic errors and the errors arising from an inefficient electrostatic correction happen to produce an almost perfect $1 / N^{2}$ behavior in the practically accessible region. To shed some more light on this, we estimated the electrostatic correction error of the standard scheme beyond the range accessible by DFT. For this, we compared electrostatic model predictions of the tuned lateral broadened setting with those of the default ones, and added them to our converged value of $4.79 \mathrm{eV}$ to predict how the plot would develop for larger cell sizes up to $200 \times 200$ (blue line). It clearly shows a nonlinear behavior in $N^{-2}$, making it problematic to extrapolate from only a few values. While the magnitude of the extrapolation error is very small in this case $(\sim 5 \mathrm{meV})$ and even within the numerical noise limit, we again observe that there is no improvement compared to using one of the rather small cells (here: $6 \times 6$ ) in combination with a proper physical modeling.

\section{CONCLUSIONS}

In summary, we have shown for two simple prototypical scenarios, namely, the ubiquitous wave-function overlap re- lated errors and charged-defect interactions in slab systems, that finite-size errors do not generally exhibit a simple global polynomial behavior as a function of the inverse supercell size. This does not really come as a surprise in view of the physical background of these effects. In consequence, scaling extrapolation does not systematically improve the accuracy of the results beyond the best explicitly calculated value if the underlying physical mechanism is not taken into consideration or not even clear.

Taking a charged defect in a 2D material for illustration, we have then shown that the combined nonlinear effects may sometimes produce simple-looking linear trends. By tracing the physical origin of the dominant error to an insufficient modeling of anisotropic charge distributions, accurate results could be obtained from much smaller cell sizes.

Our findings question the implicit assumption underlying "supercell extrapolation," that it would be an efficient "poorman's solution" to estimate finite-size errors of unspecified origin. Our results highlight that the good quality of the fits obtained from typical supercell ranges has no significance for the accuracy of the extrapolated value, and that there are cases where extrapolation magnifies errors that show an inherently different scaling behavior than implicitly assumed. Whenever possible, physically motivated correction schemes should be preferred.

\section{APPENDIX A: ONE-DIMENSIONAL TIGHT-BINDING MODEL}

To demonstrate the supercell size dependence of defect states, we work with a simple tight-binding model in one dimension and periodic boundary conditions with a single band in the unit cell. The host "bulk" material is characterized by an onsite energy $\epsilon_{b}$ and a hopping-matrix element $t_{b}$. The model Hamiltonian matrix of a periodic supercell of $N$ sites $(N \geqslant 3)$ will then read as

$$
\begin{aligned}
H_{i, i}^{k} & =\epsilon_{b}, \\
H_{i, i \pm 1}^{k} & =t_{b} \quad \text { if } 1 \leqslant i \pm 1 \leqslant N, \\
H_{1, N}^{k} & =t_{b} e^{i k N}, \\
H_{N, 1}^{k} & =t_{b} e^{-i k N}
\end{aligned}
$$

for the nonzero matrix elements $(i=1 \ldots N)$, and $H_{i j}=0$ otherwise. Here, $k$ is the one-dimensional Bloch "vector," that characterizes the phase relation upon translations. The eigenvalues of this bulk Hamiltonian are $(n=1 \ldots N)$

$$
\epsilon_{n}(k)=\epsilon_{b}+2 t_{b} \cos \left[|k|+\Delta k_{n}\right]
$$

within the first Brillouin zone $\left(-\frac{\pi}{N} \leqslant k \leqslant \frac{\pi}{N}\right)$ where

$$
\Delta k_{n}=\frac{\pi}{2 N}\left[(-1)^{n-1}(2 n-1)-1\right] .
$$

This corresponds to the backfolded band structure of the single-site case $(N=1)$

$$
\epsilon(k)=H_{11}^{k}=\epsilon_{b}+t_{b} e^{i k}+t_{b} e^{-i k}=\epsilon_{b}+2 t_{b} \cos k .
$$

The full bandwidth is $4 t_{b}$. We note in passing that changing the sign of the hopping matrix corresponds to a trivial phase 
shift in the band structure

$$
\epsilon_{-t_{b}}(k)=\epsilon_{+t_{b}}(k+\pi)
$$

which does not affect the subsequent conclusions. We will therefore assume $t_{b}>0$. Moreover, since $\epsilon_{b}$ trivially shifts the entire band structure, we set $\epsilon_{b}=-2 t_{b}$ such that the top of the bulk band is at energy zero.

To introduce a defect at site $i=2$, we modify the onsite matrix element as

$$
H_{2,2}^{k}=\epsilon_{d}
$$

and the hopping matrix elements as

$$
\begin{aligned}
& H_{1,2}^{k}=H_{2,1}^{k}=t_{d}^{-} \quad \text { and } \\
& H_{2,3}^{k}=H_{3,2}^{k}=t_{d}^{+} .
\end{aligned}
$$

The ratio $t_{d}^{+} / t_{d}^{-}$can be used to tune the symmetry of the defect, but we restrict ourselves here to the symmetric case $t_{d}^{+}=t_{d}^{-}=t_{d}$.

In the limit $N \rightarrow \infty$, and given suitably chosen parameters, this modification introduces a defect state at $E_{d}>0$ given implicitly by

$$
E_{d}=\epsilon_{d}+\frac{2 t_{d}^{2}}{t_{b}} e^{-\gamma}
$$

and

$$
\cosh \gamma=\frac{E_{d}-\epsilon_{b}}{2 t_{b}} .
$$

In practice, we choose the isolated defect state energy $E_{d}>0$ to get

$$
\gamma=\operatorname{acosh} \frac{E_{d}-\epsilon_{b}}{2 t_{b}}
$$

and then obtain $\epsilon_{d}$ from Eq. (A8).

The defect state $\mathbf{c}$ resulting from

$$
\mathbf{H}^{k} \mathbf{c}^{k}=E_{d}^{k} \mathbf{c}^{k}
$$

in the isolated-defect limit $(N \rightarrow \infty, k \rightarrow 0$, and site index $i=-\infty \ldots \infty)$ is exponentially localized, and decays like $e^{-\gamma|i-2|}$ away from the defect site, specifically,

$$
c_{i}= \begin{cases}\tilde{c}_{2} e^{-\gamma(i-2)} & \text { for } i>2, \\ \tilde{c}_{2} e^{+\gamma(i-2)} & \text { for } i<2 .\end{cases}
$$

The wave function at the defect site $i=2$ is

$$
c_{2}=\frac{t_{b}}{t_{d}} \tilde{c}_{2}
$$

and $\tilde{c}_{2}$ is given from the normalization condition as

$$
\tilde{c}_{2}=\left[\frac{2 e^{-2 \gamma}}{1-e^{-2 \gamma}}+\frac{t_{b}^{2}}{t_{d}^{2}}\right]^{-1 / 2} .
$$

This explicit eigenvector also sheds light on the qualitative role of the ratio $t_{d} / t_{b}$ : In the limit $t_{d} \rightarrow 0$, the defect state gets fully localized on the defective site at $i=2$, giving vanishing weight to the defect tails. If $t_{d}$ gets large, most of the weight of the defect state above the host band $\left(E_{d}>0\right)$ will be in the exponential tails. For sufficiently large couplings $t_{d} \gtrsim$ $\left(E_{d}+2 t_{b}\right) / \sqrt{2}$, a second defect-related deep state develops near $\epsilon_{d}<-4\left|t_{b}\right|$ below the host band with most weight on the defect site itself.

\section{APPENDIX B: WAVE-FUNCTION OVERLAP ERRORS FROM TIGHT BINDING}

The connection between the band dispersion, the k-point sampling, and the total energy in DFT can be estimated to first order from the Harris-Foulkes functional [36,37]

$$
E^{\mathrm{tot}}=\sum_{n \mathbf{k}} w_{\mathbf{k}} f_{n \mathbf{k}} \epsilon_{n \mathbf{k}}+E_{\mathrm{dc}} .
$$

Here, $f_{n \mathbf{k}}$ is the occupation number of band $n$ at some $\mathbf{k}$ point, $\epsilon_{n \mathbf{k}}$ the corresponding energy, $w_{\mathbf{k}}$ the corresponding Brillouin zone summation weight, and $E_{\mathrm{dc}}$ the double-counting correction which depends on the electron density in the DFT formalism. The defect formation energy in our model then becomes

$$
\begin{aligned}
E^{f}= & \sum_{n \mathbf{k}} w_{\mathbf{k}}\left(f_{n \mathbf{k}} \epsilon_{n \mathbf{k}}^{\text {bulk+defect }}-f_{b} \epsilon_{n \mathbf{k}}^{\text {bulk }}\right) \\
& +\underbrace{E_{\mathrm{dc}}^{\mathrm{bulk}+\text { defect }}-E_{\mathrm{dc}}^{\mathrm{bulk}}}_{=E_{\mathrm{dc}}^{f}} .
\end{aligned}
$$

$f_{b}$ is the occupation number of the bulk bands. Equation (B2) can be simplified as follows. The sum of all eigenvalues is the trace of the Hamiltonian matrix $\mathbf{H}^{\mathbf{k}}$, which is $N \epsilon_{b}$ for the pure bulk with $N$ sites in the supercell. For the defect,

$$
\sum_{n} \epsilon_{n \mathbf{k}}=\operatorname{tr} \mathbf{H}^{\mathbf{k}}=N \epsilon_{b}+\left(\epsilon_{d}-\epsilon_{b}\right)
$$

is independent of $\mathbf{k}$. We will further assume that all bands except for the differently occupied defect band $n=d$ have full bulk occupation (if this is not the case, significant errors must be expected). With this background, Eq. (B2) can be rewritten as

$$
\begin{aligned}
E^{f}= & E_{\mathrm{dc}}^{f}+\sum_{n \mathbf{k}} w_{\mathbf{k}}\left(f_{n \mathbf{k}}-f_{b}\right) \epsilon_{n \mathbf{k}}^{\text {bulk }+ \text { defect }} \\
& +\sum_{n \mathbf{k}} w_{\mathbf{k}} f_{b}\left(\epsilon_{n \mathbf{k}}^{\text {bulk+defect }}-\epsilon_{n \mathbf{k}}^{\text {bulk }}\right) \\
= & \sum_{\mathbf{k}} w_{\mathbf{k}}\left(f_{d \mathbf{k}}-f_{b}\right) \epsilon_{d \mathbf{k}}^{\text {bulk+defect }} \\
& +E_{\mathrm{dc}}^{f}+f_{b}\left(\epsilon_{d}-\epsilon_{b}\right) .
\end{aligned}
$$

The finite-size error in the defect formation energy then is

$$
\Delta E^{f}=\sum_{\mathbf{k}} w_{\mathbf{k}}\left(f_{d \mathbf{k}}-f_{b}\right)\left(\epsilon_{d \mathbf{k}}-E_{d}\right)+\Delta E_{\mathrm{dc}}^{f},
$$

where $\Delta E_{\mathrm{dc}}^{f}$ denotes the error in the double-counting term.

Equation (B5) implies that supercell errors arising from wave-function overlap must be expected whenever a defect band couples to a bulk band with a different occupation $f_{d \mathbf{k}} \neq$ $f_{b}$, and when the occupation-weighted average of the defect state energy over the Brillouin zone does not correspond to the isolated defect state's energy. While this isolated value is generally not known in actual DFT calculations, our model allows us to discuss qualitatively the impact of different choices for the k-point sampling and occupation scheme. Independent of these choices, as shown in Sec. IIIC, the error decays exponentially with supercell size. 
The density-related term in Eq. (B5), $\Delta E_{\mathrm{dc}}^{f}$, probably shows an exponential convergence by itself because the defect-induced density is coupled to the exponentially decaying defect states. However, we failed to verify this numerically because the double-counting term in DFT depends on the potential alignment (equivalent to affecting both $\epsilon_{d}$ and $\epsilon_{b}$ ), and even small uncertainties in the alignment $(\Delta V$, on the order of a few $0.01 \mathrm{eV}$ ) produce very large uncertainties in the doublecounting energy differences $\Delta E_{\mathrm{dc}}=N_{e} \Delta V$ of large supercells with several hundred electrons $\left(N_{e}\right)$. In lack of further insight into this term, we will not discuss this contribution any further, but expect that it does not compensate systematically for the $\epsilon_{d \mathbf{k}}$-related convergence.

\section{APPENDIX C: SCALING OF ELASTIC INTERACTIONS}

Elastic interactions between point defects in bulk supercells are known to scale as $L^{-3}[4,38]$. The scaling in 2D materials has to our knowledge not been discussed before, but can easily be shown to be $L^{-2}$ as follows.

The displacement $\mathbf{u}(s, \mathbf{R})$ of an atom at site $s$ in lattice cell $\mathbf{R}$ induced by a defect can be obtained from the Kanzaki forces $\mathbf{f}\left(s^{\prime}, \mathbf{R}^{\prime}\right)$ at site $s^{\prime}$ in cell $\mathbf{R}^{\prime}$ via $[38,39]$

$$
u_{\alpha}(s, \mathbf{R})=\sum_{s^{\prime}, \beta} \mathcal{G}_{\alpha \beta}^{s s^{\prime}}\left(\mathbf{R}-\mathbf{R}^{\prime}\right) f_{\beta}\left(s^{\prime}, \mathbf{R}^{\prime}\right)
$$

using the bulk's elastic Green's function $\mathcal{G}_{\alpha \beta}^{s{ }^{\prime}}$. Fourier transformation to reciprocal space yields

$$
u_{\alpha}(s, \mathbf{k})=\sum_{s^{\prime}} \mathcal{G}_{\alpha \beta}^{s s^{\prime}}(\mathbf{k}) f_{\beta}\left(s^{\prime}, \mathbf{k}\right) .
$$

The elastic energy is then given by [39]

$$
E_{\mathrm{el}}^{N_{\mathbf{k}}}=-\frac{1}{2 N_{\mathbf{k}}} \sum_{s, \mathbf{k} \neq 0} \mathbf{u}^{*}(s, \mathbf{k}) \cdot \mathbf{f}(s, \mathbf{k}),
$$

where the sum over $\mathbf{k}$ runs over the nonzero reciprocal lattice vectors of the supercell that are inside the Brillouin zone of the underlying bulk lattice. $N_{\mathbf{k}}$ is the number of all supercell lattice vectors inside the Brillouin zone of the underlying lattice, and hence equivalent to the number of unit cells in the supercell $N_{\mathrm{sc}}$. The omission of the $\mathbf{k}=0$ term corresponds imposing zero strain, i.e., using the supercell with the bulk lattice constants.

In the limit of large supercells, the sum transforms into the integral

$$
E_{\mathrm{el}}^{\infty}=-\frac{1}{2 \Omega_{\mathrm{BZ}}} \int_{\mathrm{BZ}} d^{D} \mathbf{k} \mathbf{u}^{*}(\mathbf{k}) \cdot \mathbf{f}(\mathbf{k}) .
$$

Here, $\Omega_{\mathrm{BZ}}$ denotes the "volume" of the bulk lattices Brillouin zone in $D$ dimensions (i.e., an area for $D=2$ ). The integrand is a smooth function except at $\mathbf{k}=0$, where it is nonanalytic. For this, note that $\mathbf{f}(\mathbf{k}) \sim|\mathbf{k}|, \mathcal{G}(\mathbf{k}) \sim 1 /|\mathbf{k}|^{2}$, and hence $\mathbf{u}(\mathbf{k}) \sim 1 /|\mathbf{k}|$ independent of the dimensionality $[39,40]$. In consequence, the integrand remains finite near $\mathbf{k}=0$, but it may vary with the direction $\hat{\mathbf{k}}=\mathbf{k} /|\mathbf{k}|$, i.e., $\mathbf{u}(\mathbf{k}) \cdot f(\mathbf{k}) \rightarrow$ $\eta(\hat{\mathbf{k}})$.

The leading error when comparing the integral with the finite sum arises from entirely omitting the $\mathbf{k}=0$ term in the finite sum. A better approximation would be to replace it by the analytic integral over the Brillouin zone of the supercell (scBZ),

$$
\Delta E_{\mathbf{k}=0}(\operatorname{scBZ}) \approx \int_{\operatorname{scBZ}} d^{D} \mathbf{k} \eta(\mathbf{k} /|\mathbf{k}|) .
$$

As the integrand does not depend on the length scale, the integral factorizes into a volume factor $\Omega_{\mathrm{scBZ}}$ and an angular factor $A$ that depends on the ratio of the superlattice vectors $\mathbf{a}_{1}^{\mathrm{sc}}: \mathbf{a}_{2}^{\mathrm{sc}}: \mathbf{a}_{3}^{\mathrm{sc}}$ of the supercell and the intrinsic orientational dependence of $\eta$. Hence, for a given superlattice ratio,

$$
\begin{aligned}
\Delta E_{\mathbf{k}=0}(\mathrm{BZ}, \mathrm{sc}) & \approx \frac{1}{2} \Omega_{\mathrm{scBZ}} A\left(\mathbf{a}_{1}^{\mathrm{sc}}: \mathbf{a}_{2}^{\mathrm{sc}}: \mathbf{a}_{3}^{\mathrm{sc}}, \eta\right) \\
& =\frac{1}{2} \frac{\Omega_{\mathrm{BZ}}}{N_{\mathrm{sc}}} A\left(\mathbf{a}_{1}^{\mathrm{sc}}: \mathbf{a}_{2}^{\mathrm{sc}}: \mathbf{a}_{3}^{\mathrm{sc}}, \eta\right) .
\end{aligned}
$$

For a uniform scaling of a $D$-dimensional supercell (e.g., $N \times$ $N \times N$ for $D=3, N \times N$ for $D=2, N$ for $D=1$, and $N_{\text {sc }}=$ $N^{D}$ ), this is equivalent to a $L^{-D}$ scaling since $L_{N}=N L_{1}$.
[1] R. J. Maurer, C. Freysoldt, A. M. Reilly, J. G. Brandenburg, O. T. Hofmann, T. Björkman, S. Lebègue, and A. Tkatchenko, Annu. Rev. Mater. Res. 49, 1 (2019).

[2] S. G. Louie, M. Schlüter, and J. R. Chelikowsky, Phys. Rev. B 13, 1654 (1976).

[3] R. M. Nieminen, in Theory of Defects in Semiconductors, edited by D. A. Drabold and S. K. Estreicher, Topics in Applied Physics Vol. 104, (Springer, Berlin, 2007), p. 29.

[4] C. Freysoldt, B. Grabowski, T. Hickel, J. Neugebauer, G. Kresse, A. Janotti, and C. G. Van de Walle, Rev. Mod. Phys. 86, 253 (2014).

[5] J. Spitaler and S. K. Estreicher, Front. Mater. 5, 70 (2018).

[6] C. G. Van de Walle and J. Neugebauer, J. Appl. Phys. 95, 3851 (2004).

[7] G. Makov, R. Shah, and M. C. Payne, Phys. Rev. B 53, 15513 (1996).

[8] G. Makov and M. C. Payne, Phys. Rev. B 51, 4014 (1995).
[9] C. Freysoldt, J. Neugebauer, and C. G. Van de Walle, Phys. Rev. Lett. 102, 016402 (2009).

[10] C. W. M. Castleton, A. Höglund, and S. Mirbt, Phys. Rev. B 73, 035215 (2006).

[11] P. A. Schultz, Phys. Rev. Lett. 96, 246401 (2006).

[12] A. F. Wright and N. A. Modine, Phys. Rev. B 74, 235209 (2006).

[13] R. Nieminen, Modelling Simul. Mater. Sci. Eng. 17, 084001 (2009).

[14] S. Lany and A. Zunger, Phys. Rev. B 78, 235104 (2008).

[15] Y. Kumagai and F. Oba, Phys. Rev. B 89, 195205 (2014).

[16] Z.-J. Suo, J.-W. Luo, S.-S. Li, and L.-W. Wang, Phys. Rev. B 102, 174110 (2020).

[17] M. Chagas da Silva, M. Lorke, B. Aradi, M. Farzalipour Tabriz, T. Frauenheim, A. Rubio, D. Rocca, and P. Deák, Phys. Rev. Lett. 126, 076401 (2021). 
[18] A. Walsh, npj Comput. Mater. 7, 72 (2021).

[19] H.-P. Komsa and A. Pasquarello, Phys. Rev. Lett. 110, 095505 (2013).

[20] H.-P. Komsa, N. Berseneva, A. V. Krasheninnikov, and R. M. Nieminen, Phys. Rev. X 4, 031044 (2014).

[21] R. Sundararaman and Y. Ping, J. Chem. Phys. 146, 104109 (2017).

[22] C. Freysoldt and J. Neugebauer, Phys. Rev. B 97, 205425 (2018).

[23] M. H. Naik and M. Jain, Comput. Phys. Commun. 226, 114 (2018).

[24] T. Gake, Y. Kumagai, C. Freysoldt, and F. Oba, Phys. Rev. B 101, 020102(R) (2020).

[25] S. Falletta, J. Wiktor, and A. Pasquarello, Phys. Rev. B 102, 041115(R) (2020).

[26] C. Varvenne, F. Bruneval, M.-C. Marinica, and E. Clouet, Phys. Rev. B 88, 134102 (2013).

[27] J. Shim, E.-K. Lee, Y. J. Lee, and R. M. Nieminen, Phys. Rev. B 71, 035206 (2005).

[28] https://sxrepo.mpie.de.
[29] S. Boeck, C. Freysoldt, A. Dick, L. Ismer, and J. Neugebauer, Comput. Phys. Commun. 182, 543 (2011).

[30] G. Schwarz, Untersuchungen zu Defekten auf und nahe der (110)-Oberfläche von GaAs und weiteren III-V-Halbleitern, Ph.D. thesis, Technical University Berlin, 2001.

[31] C. A. Rozzi, D. Varsano, A. Marini, E. K. U. Gross, and A. Rubio, Phys. Rev. B 73, 205119 (2006).

[32] S. Ismail-Beigi, Phys. Rev. B 73, 233103 (2006).

[33] H.-P. Komsa, N. Berseneva, A. V. Krasheninnikov, and R. M. Nieminen, Phys. Rev. X 8, 039902(E) (2018).

[34] C. Freysoldt, P. Eggert, P. Rinke, A. Schindlmayr, and M. Scheffler, Phys. Rev. B 77, 235428 (2008).

[35] https://sxrepo.mpie.de/projects/sphinx-add-ons/files.

[36] J. Harris, Phys. Rev. B 31, 1770 (1985).

[37] W. M. C. Foulkes and R. Haydock, Phys. Rev. B 39, 12520 (1989).

[38] E. Clouet, C. Varvenne, and T. Jourdan, Comput. Mater. Sci. 147, 49 (2018).

[39] V. K. Tewary, Adv. Phys. 22, 757 (1973).

[40] D. R. Trinkle, Phys. Rev. B 78, 014110 (2008). 